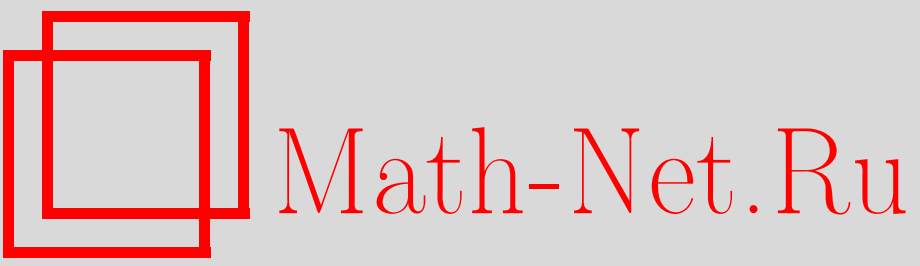

Л. В. Розовский, Об условиях сходимости обобщенного ряда Спицера, Теория вероятн. и ее примен., 2012, том 57, выпуск 4, 809-810

DOI: https://doi.org/10.4213/tvp4484

Использование Общероссийского математического портала Math-Net.Ru подразумевает, что вы прочитали и согласны с пользовательским соглашением

http://www.mathnet.ru/rus/agreement

Параметры загрузки:

IP : 54.224 .60 .19

26 апреля 2023 г., 14:18:56 
3. Курбанмурадов О. Методы блуждания по шароидам для решения уравнения теплопроводности. - Теория и алгоритмы статистического моделирования. Новосибирск: ВЦ СО АН СССР, 1984, с. 67-77.

4. Ладыженская О.А., Солонников В.А., Уральчева Н. Н. Линейные и квазилинейные уравнения параболического типа. М.: Наука, 1967, 736 с.

5. Лионс Ж.-Л. Оптимальное управление системами, описываемыми уравнениями с частными производными. М.: Мир, 1972, 414 с.

6. Михайлов Г.А. Оптимизация весовых методов Монте-Карло. М.: Наука, 1987, $239 \mathrm{c}$.

7. Михайлов Г.А. Весовые методы Монте-Карло. Новосибирск: Изд-во СО РАН, $2000,248 \mathrm{c}$.

8. Мейер П.-А. Вероятность и потенциалы. М.: Мир, 1973, 324 с.

9. Ширяев А. Н. Вероятность. М.: Наука, 1989, 640 с.

10. Rasulov A., Mascagni M., Raimova G. Monte Carlo Methods for the Solution of Linear and Nonlinear Boundary Value Problems. Tashkent: UWED, 2006, 346 p.

Поступила в редакцию

18.VI.2009

Исправленный вариант 20.X.2012

(c) 2012 г.

РОЗОВСКИЙ Л. В.*

\section{ОБ УСЛОВИЯХ СХОДИМОСТИ ОБОБЩЕННОГО РЯДА СПИЦЕРА}

\footnotetext{
В заметке приведены оптимальные моментные условия, при которых сходится обобщенный ряд Спицера.

Ключевые слова и фразы: суммы независимых случайных величин, ряд Спицера, нормальное распределение.
}

Рассмотрим независимые одинаково распределенные случайные величины $X, X_{1}, X_{2}, \ldots$ с нулевым средним и конечной положительной дисперсией $\sigma^{2}$. Положим $S_{n}=X_{1}+\cdots+X_{n}$.

Мы приведем один результат, связанный с оценкой величины

$$
\mathbf{P}\left(S_{n}<0\right)-\frac{1}{2}
$$

а именно сформулируем оптимальные условия, при которых сходится ряд

$$
\sum_{n \geqslant 1} \frac{g(n)}{n}\left|\mathbf{P}\left(S_{n}<0\right)-\frac{1}{2}\right|,
$$

где положительные веса $g(n)$ имеют степенной характер.

При $g(n) \equiv 1$ ряд из (1) аналогичен известному ряду Спицера, оценка сверху для суммы которого была недавно получена в [1].

* С.-Петербургская химико-фармацевтическая академия, кафедра высшей математики, ул. проф. Попова, 14, 197376 С.-Петербург, Россия; e-mail: l_rozovski@mail.ru

1) Исследование выполнено при поддержке программы «Ведущие научные школы» (грант НШ 638.2008.1) и РФФИ 10-01-00242-а. 
Теорема. Пусть положительная функиия $g(x)$ при всех $x \geqslant 0$ и при некоторьх $\delta<1$ и $\alpha<1 / 2$ удовлетворяет условиям

$$
g(x) x^{-\delta} \quad \text { не возрастает, } g(x) x^{\alpha} \quad \text { не убъввает, }
$$

u, кроме того, $\int_{1}^{\infty} g(x) x^{-3 / 2} d x<\infty$. Если $\mathbf{E} X^{2} g\left(X^{2}\right)<\infty$, то ряд в (1) сходится.

Утверждение теоремы вытекает их нижеследующей леммы, которая, в свою очередь, является частным случаем теоремы 7 из [2].

Лемма. Положим $D_{n}=\mathbf{E} X^{2} \mathbf{I}\left[|X|<\sqrt{n}, b_{n}=\sqrt{n D_{n}}\right.$. В условиях теоремь

$$
\sum_{n \geqslant 1} \frac{g(n)}{n} \Delta_{n}<\infty
$$

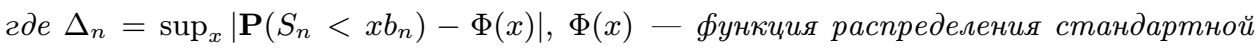
нормальной случайной величинь.

В заключение покажем, что утверждение, сделанное в [1, Замечание] о том, что

$$
I=\sum_{n \geqslant 1} n^{-1}\left|\mathbf{P}\left(S_{n}<x \sigma \sqrt{n}\right)-\Phi(x)\right|<\infty
$$

вообще говоря, не верно при любом $x \neq 0$.

Пусть $\sigma^{2}=1$ и, для определенности, $x>0$. Тогда

$$
\begin{aligned}
\left|\mathbf{P}\left(S_{n}<x \sqrt{n}\right)-\Phi(x)\right| & \geqslant\left|\Phi\left(\frac{x \sqrt{n}}{b_{n}}\right)-\Phi(x)\right|-\left|\mathbf{P}\left(S_{n}<\left(\frac{x \sqrt{n}}{b_{n}}\right) b_{n}\right)-\Phi\left(\frac{x \sqrt{n}}{b_{n}}\right)\right| \\
& \geqslant \Phi\left(\frac{x \sqrt{n}}{b_{n}}\right)-\Phi(x)-\Delta_{n} .
\end{aligned}
$$

Очевидно,

$$
\Phi\left(\frac{x \sqrt{n}}{b_{n}}\right)-\Phi(x) \sim \frac{1}{2} x \Phi^{\prime}(x)\left(\frac{n}{b_{n}^{2}}-1\right) \sim \frac{x \Phi^{\prime}(x)}{2}\left(1-D_{n}\right), \quad n \rightarrow \infty .
$$

Таким образом, при любом фиксированном $x>0$

$$
I+\sum_{n \geqslant 1} n^{-1} \Delta_{n} \geqslant c \sum_{n \geqslant 1} n^{-1}\left(1-D_{n}\right)
$$

где $c$ - некоторая положительная постоянная. По лемме ряд слева в (4) сходится, в то время как сходимость ряда справа равносильна существованию момента $\mathbf{E} X^{2} \ln (1+|X|)$. Иначе ряд $I$ расходится.

\section{СПИСОК ЛИТЕРАТУРЫ}

1. Нагаев A. В. Новое доказательство абсолютной сходимости ряда Спицера. - Теория вероятн. и ее примен., 2009, т. 54, в. 1, с. 149-152.

2. Розовский Л. В. О точности оценки остаточного члена в центральной предельной теореме. - Теория вероятн. и ее примен., 1978, т. 23, в. 4, с. 744-761.

Поступила в редакцию 